\title{
Tracking challenges of QKD over relay satellite
}

\section{SPIE.}

\author{
S. Sharma, N. Perlot, J. Rödiger, R. Freund
}

Fraunhofer Heinrich Hertz Institute, Einsteinufer 37, 10587 Berlin, Germany

\section{Fraunhofer}

Heinrich Hertz Institute

\begin{tabular}{|c|c|c|c|c|}
\hline \multicolumn{5}{|c|}{ What about QKD over relay satellite? } \\
\hline \multicolumn{5}{|c|}{$\begin{array}{l}\text { We analyze quantum key distribution (QKD) with a satellite acting as a relay node between } \\
\text { two distant Earth-based parties, Alice and Bob (last scenario in Table 1). } \\
\text { Table 1. Four QKD scenarios involving a satellite. }\end{array}$} \\
\hline $\begin{array}{c}\text { Must satellite be } \\
\text { trusted? }\end{array}$ & Yes & & No & \\
\hline Satellite role & $\begin{array}{l}\text { One-way } \\
\text { distributor }\end{array}$ & $\begin{array}{c}\text { Entangled photon } \\
\text { source }\end{array}$ & $\begin{array}{l}\text { Bell-measurement } \\
\text { station }\end{array}$ & Relay mirror \\
\hline Depiction & $\underset{\text { Alice }}{\downarrow}$ & Alice Bob & $\prod_{\text {Bob }}$ & $\Gamma_{\text {Blice }}$ \\
\hline QKD principle & $\begin{array}{c}\text { Prepare and } \\
\text { measure }\end{array}$ & $\begin{array}{c}\text { Entanglement } \\
\text { based }\end{array}$ & $\begin{array}{c}\text { Measurement- } \\
\text { device independent }\end{array}$ & $\begin{array}{c}\text { Prepare and } \\
\text { measure }\end{array}$ \\
\hline
\end{tabular}

\section{Point-ahead angles (PAA)}

Satellite speed requires beams to be pointed "ahead" of the received beams.

- If Alice's PAA strongly differs from Bob's PAA $\rightarrow$ relay mirror cannot maintain bidirectional transmission.

" The difference between the PAA vectors $(\Delta \theta)$ must be evaluated.

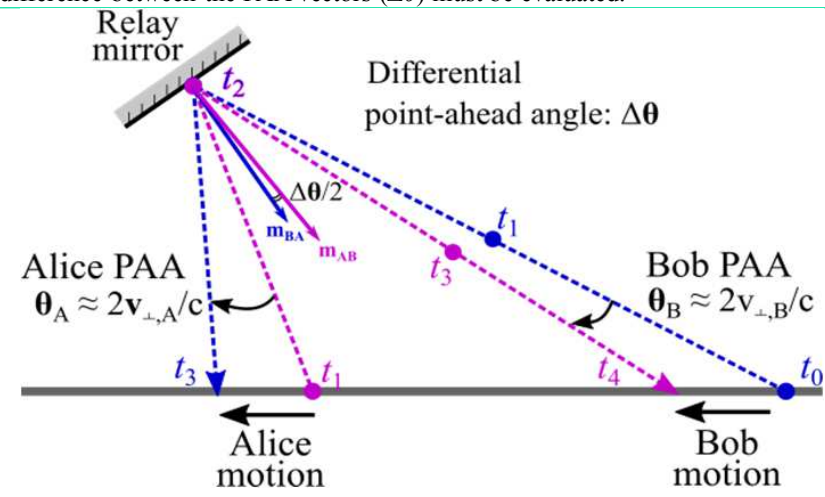

Fig. 1: Rays sent and received by Alice and Bob from the viewpoint of the flying mirror at different times $t_{N}$. The point-ahead angle $\boldsymbol{\theta}_{\mathrm{A}}$ (resp. $\boldsymbol{\theta}_{\mathrm{B}}$ ) on Alice's side (resp. Bob's side) depends on the beamtransverse velocity $\mathbf{v}_{\perp, \mathrm{A}}\left(\right.$ resp. $\left.\mathbf{v}_{\perp, \mathrm{B}}\right)$ of Alice (resp. Bob) and on the light velocity c. Generally, $\theta_{A} \neq \theta_{B} \cdot \mathbf{m}_{\mathrm{AB}}$ and $\mathbf{m}_{\mathrm{B} A}$ are the mirror vectors required to satisfy Alice-mirror-Bob link and Bobmirror-Alice link respectively.

- We consider a LEO satellite (altitude $500 \mathrm{~km}$, speed $7.6 \mathrm{~km} / \mathrm{s}$ ) moving from south to north - We analyze the variation of $\Delta \theta$ of the two ground stations, $1000 \mathrm{~km}$ apart from each other for two cases of interest.

- PAA magnitude is shown in Fig. 2 (right) and variation of $\Delta \theta$ is shown in Fig. 3.
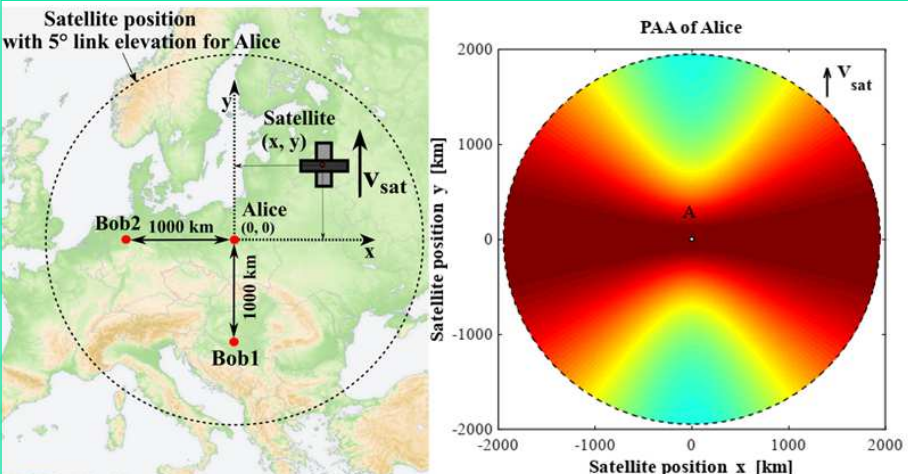

[ $\mu \mathrm{rad}]$

Fig. 2: Considered geometry of Alice, Bob and the satellite (left). PAA of the Alice-satellite link along the satellite's Earth-surface coordinates $(x, y)$ (right).
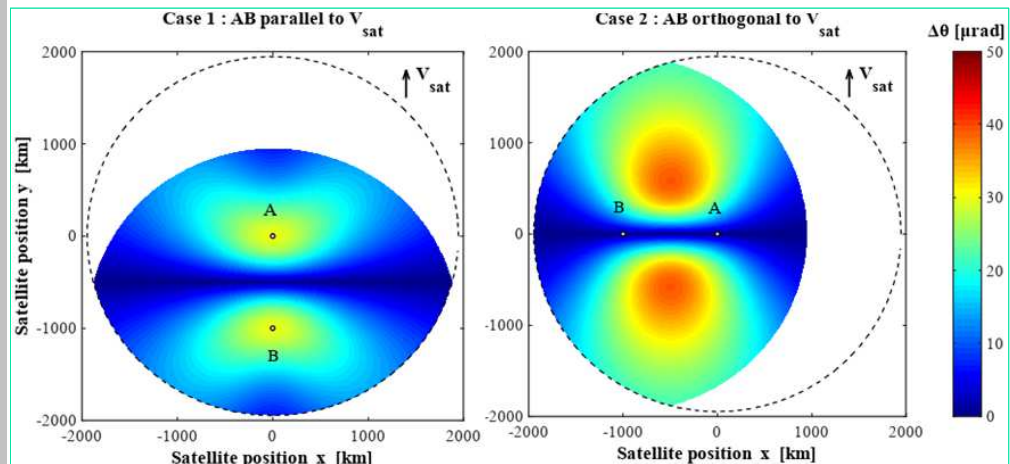

Fig. 3: Variation of the difference in PAA of Alice and Bob along the coordinates of the satellite $(\mathrm{x}, \mathrm{y})$ with Alice as the origin when $A B$ parallel to $\mathbf{V}_{\text {sat }}$ (left) and when $A B$ orthogonal to $\mathbf{V}_{\text {sat }}$ (right).

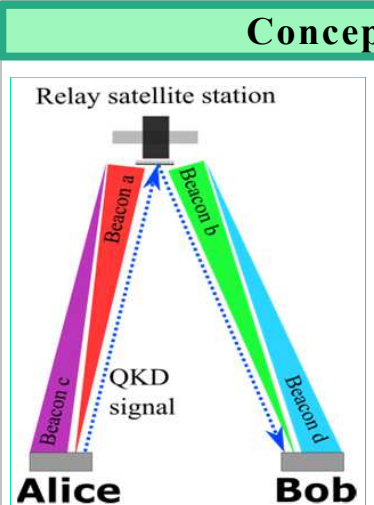

Fig. 4: Considered be
and relay OKD beam.
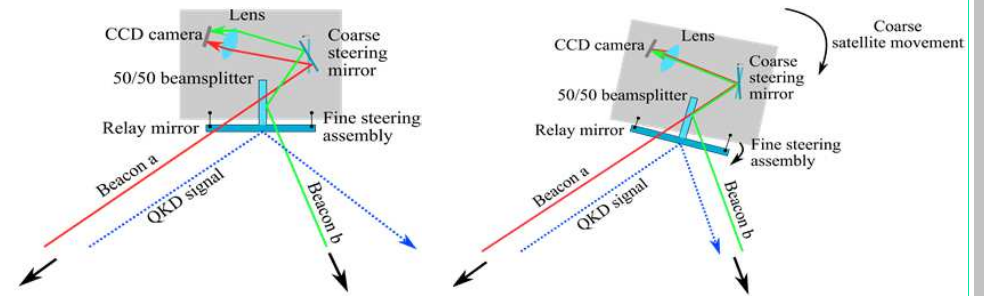

Fig. 5: Proposed fine tracking system of the relay-satellite station for the case of large inciden angles, where the beam splitter and relay mirror constitute a monolithic assembly. Assuming negligible PAA, correct pointing is achieved when the two spots of Beacon a and Beacon b superpose on the CCD camera. A coarse steering mirror keeps the two beams on a CCD camera. With a significant differential PAA, the two spots should no longer superpose, but be dissociated with respect to the differential-PAA vector $\Delta \boldsymbol{\theta}$.

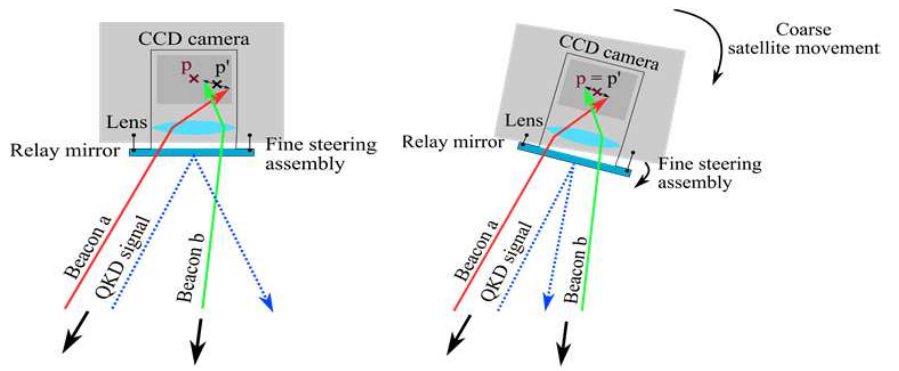

Fig. 6: Proposed fine tracking system of the relay-satellite station for the case of smaller inciden angles. Center of Beacon a and Beacon $\mathrm{b}$ (marked as $\mathrm{p}^{\prime}$ ) should be moved to point of reference ( $\mathrm{p}$ ) which is the relay mirror's direction corrected by PAA offset for accurate pointing of the signal.

\section{Link power budget}

With a symmetric up and down propagation path and ignoring the atmospheric losses, we obtain a rough and optimistic link loss of $\sim 40 \mathrm{~dB}$.

Propagation loss double of what we expect from a trusted node scenario.

- Uplink is more challenging than downlink because atmospheric turbulence cannot be fully compensated.

A non-symmetrical relay link is expected in the presence of uncompensated turbulence.

Considering telescope diameter of Alice ground station $\approx 0.3 \mathrm{~m}$ while for Bob ground station $\sim 1 \mathrm{~m}$, we obtain a link loss of around $50 \mathrm{~dB}$

\section{Conclusion}

Not so simple, but still attractive.

Complex tracking system required to point the mirror in the correct direction.

- Large (differential) PAAs,

- Several onboard acquisition systems (beacon measurements).

Higher propagation loss than the trusted node satellite QKD.

No need to trust the satellite.

Alice and Bob on ground, no complicated sources or receivers on the satellite.

Potential to provide higher key rates than the entanglement-based or MDI QKD.

Offers flexibility to choose any protocol and wavelength.
Sakshi.sharma@hhi.fraunhofer.de www.hhi.fraunhofer.de 